\title{
Study of psychological health of healthy volunteers by symptom checklist 90 (SCL-90) and eysenck personality questionnaire (EPQ).
}

\author{
Yudong Wei ${ }^{1 *}$, Haiyan $\mathrm{Li}^{2}$, Huali Wang ${ }^{3}$, Shuang Zhang1, Yumei Sun ${ }^{4 *}$ \\ ${ }^{1}$ Drug Clinical Trial Center, Peking University Third Hospital, 49 North Garden Road, Haidian District, Beijing, PR China \\ ${ }^{2}$ The Department of Cardiology, Peking University Third Hospital, 49 North Garden Road, Haidian District, Beijing, \\ PR China \\ ${ }^{3}$ Peking University Sixth Hospital, Institute of Mental Health, 51 North Garden Road, Haidian District, Beijing, PR China \\ ${ }^{4}$ Peking University School of Nursing, 38 Xueyuan Road, Haidian District, Beijing, PR China
}

\begin{abstract}
Psychological health of Chinese healthy volunteers was investigated to provide a theoretical method for phase I clinical trial management and result analysis. Symptom checklist 90 (SCL-90) and Eysenck Personality Questionnaire (EPQ) were used to assess 200 healthy volunteers and to analyse the level of psychological health of Chinese healthy volunteers. SCL-90 result indicates that the average value of positive factors is $10.32 \pm \mathbf{1 4 . 2 6}$ by self-assessment of healthy volunteers, somatization factor is $1.13 \pm$ 0.13, compulsive symptom factor is $1.29 \pm 0.27$, interpersonal sensitivity factor is $1.31 \pm 0.21$, depression factor is $1.26 \pm 0.33$, anxiety factor is $1.21 \pm 0.21$, hostility factor is $1.08 \pm 0.26$, phobia factor is $1.05 \pm$ 0.18, paranoid factor is $1.12 \pm 0.23$, psychotic symptom factor is $1.17 \pm 0.26$. Comparing the norm in China, the score of each factor of healthy volunteers is relatively low with statistically significant difference $(P<0.001)$. EPQ result shows that $P$ score is $4.59 \pm 2.33$, $E$ score is $13.13 \pm 4.32$, $\mathrm{N}$ score is 6.89 \pm 5.26, and $L$ score is $13.21 \pm 4.25$ for 200 healthy volunteers. Comparing the norm in China, the $P$ and $\mathbf{N}$ scores are lower, and the $\mathbf{E}$ and $\mathrm{L}$ scores are higher, with statistically significant difference $(\mathbf{P}<0.001)$.
\end{abstract}

Keywords: SCL-90, EPQ, Psychological health, Phase I clinical trial.

Accepted on November 27, 2017

\section{Introduction}

Clinical research is a key step for clinical application of a new drug from laboratory. In China, clinical trials and bioequivalence tests are usually referred to as clinical studies [1]. A clinical trial can be divided into four phases in most of countries in the world, and the basic standards and technical requirements of each phase in clinical trial are strictly regulated [2]. The phase I clinical trial, which is also called clinical pharmacology and toxicology phase, aims at the new drugs which have already passed the assessment of pre-clinical safety and efficacy [3]. Normally, only healthy volunteers are involved in phase I clinical trials. In this study, the emotional qualities and personality characteristics of healthy volunteers in phase I clinical trial were investigated to understand the characteristics of volunteer. The current work provides a theoretical fundamental for the management, quality control and results analysis in future clinical studies.

\section{Objectives and Methods}

\section{Research objectives}

A convenient sampling method was applied to screen the healthy volunteers who have registered in phase I database of qualified units for drug clinical trials in a Beijing upper firstclass hospital. 200 volunteers were randomly selected by database administrator according to the registration number, the criteria include: age of 18 or above, able to communicate or write with Chinese language and voluntary participation.

\section{Methods}

Two questionnaires were used in this study-symptom checklist 90 (SCL-90) and Eysenck Personality Questionnaire (EPQ).

\section{Symptom checklist 90 (SCL-90)}

SCL-90 consists of 90 items, including a very broad area of psychiatric symptomatology ranging from feeling, emotion, thinking, consciousness, behaviors to living habits, interpersonal relationship, diet and sleep. Ten factors are used 
to reflect the psychological symptoms in ten aspects, respectively. The results of this questionnaire explain the number of positive items for primary basis (with a score of no less than 2) and score of each factor. The greater is the number of positive items, the higher the score of each factor is, which indicates that the condition of volunteer's psychological health is poor. When a score of an individual is greater than 2 (beyond the normal average number), there might be some psychological problems in that aspect. The questionnaire is a classic self-assessed list. According to Degrogatis report, the validity coefficient of various symptoms is between 0.77 and 0.99. China Scale Collaborative Group has applied Global Assessment Scale (GAS) and Social Introversion scale (SI) to check parallel validity of SCL-90, a negative correlation was found between the total score of SCL-90 and GAS $(p<0.05-0.01)$ and a positive correlation between SCL-90 score and SI $(p<0.01)$. All these results indicate that SCL-90 has good reliability and validity among the normal population. The reliability and validity meet the requirement of measurement [4].

\section{Eysenck personality questionnaire (EPQ)}

EPQ was compiled by a British psychologist H.J. Eysenck as a self-report scale, it was developed on the basis of the "Eysenck Personality Inventory" (EH) [5]. The number of items in revised version in China has changed from 107 to 88 [6], including four subscales: internal and external propensity scale $(\mathrm{E})$, neuroticism $(\mathrm{N})$, psychoticism $(\mathrm{P})$ and validity scale $(\mathrm{L})$. The reliability of $\mathrm{E}, \mathrm{N}$ and $\mathrm{L}$ scales is between 0.74 and 0.78 . However, the $\mathrm{P}$ scale reliability is between 0.54 and 0.60 , suggesting that EP makes RSC not only inherit the advantage of original questionnaire with high reliability of $\mathrm{E}, \mathrm{N}$ and $\mathrm{L}$ scale, but also retain the disadvantage of a not stable $\mathrm{P}$ scale.

\section{Data collection method}

Healthy volunteers were randomly selected by the sequence of number in a phase I clinical trial database of a qualified upper first-class hospital in Beijing, the selected volunteers were firstly contacted by phone to inform them about the purposes and procedures of study. After their agreement, they came to the drug clinical trial institution personally for a check. The staffs who take the responsibility of information collection were professionally trained to ensure that each professional staff adopts standard procedures or manners, such as using the same tone and attitude to communicate with volunteers. Thus the effect of subjective factors of researchers on information acquisition will be minimized and the subjective bias resulting from human factors will be significantly reduced. During the process of questionnaires collection, any missing items were carefully checked to ensure all questions are complete. The survey was conducted by two trained researchers with direct inquiry, one asked questions and the other recorded answers from a volunteer. A total of 200 questionnaires were issued, and 200 valid questionnaires were recovered. The recovery rate and the effective rate in this method were both $100 \%$.

\section{Statistical method}

All data were inputted using Epidata 3.0 software, SPSS 18.0 was used for statistical analysis. The continuous variables were expressed by (mean \pm standard deviation). The comparison between constituent ratios and rates was conducted using chi square test. $\mathrm{P}<0.05$ was considered as statistical significance.

\section{Results}

\section{General information}

Average age of the healthy volunteers is $28.6 \pm 7.8 \mathrm{y}$ old. Table 1 lists their age, gender, education, work, income, etc.

Table 1. General information of healthy volunteers.

\begin{tabular}{|c|c|}
\hline Items & Healthy volunteers $(n=200)$ \\
\hline \multicolumn{2}{|l|}{ Age } \\
\hline Less than 30 & 112 \\
\hline Between 30 and 44 & 88 \\
\hline Between 45 and 59 & N/A \\
\hline More than 60 & N/A \\
\hline \multicolumn{2}{|l|}{ Gender } \\
\hline Male & 146 \\
\hline Female & 54 \\
\hline \multicolumn{2}{|l|}{ Education } \\
\hline Primary school & 3 \\
\hline Junior school & 42 \\
\hline High School & 82 \\
\hline College & 55 \\
\hline University and above & 18 \\
\hline \multicolumn{2}{|l|}{ Work } \\
\hline Employed & 142 \\
\hline Unemployed & 46 \\
\hline Retired & N/A \\
\hline Housewife & N/A \\
\hline Student & 12 \\
\hline \multicolumn{2}{|l|}{ Annual income } \\
\hline Less than RMB30,000 & 48 \\
\hline Between RMB30,000 and RMB50,000 & 110 \\
\hline Between RMB50,000 and RMB70,000 & 19 \\
\hline Between RMB70,000 and RMB90,000 & 22 \\
\hline
\end{tabular}


More than 90,000 1

\section{SCL-90 results}

The average value of positive factors is $10.32 \pm 14.26$ by selfassessment of healthy volunteers. Among all factors, somatization factor is $1.13 \pm 0.13$, compulsive symptom factor is $1.29 \pm 0.27$, interpersonal sensitivity factor is $1.31 \pm 0.21$, depression factor is $1.26 \pm 0.33$, anxiety factor is $1.21 \pm 0.21$, hostility factor is $1.08 \pm 0.26$, phobia factor is $1.05 \pm 0.18$, paranoid factor is $1.12 \pm 0.23$, psychotic symptom factor is $1.17 \pm 0.26$.

Comparing the norm in China, the score of each factor of healthy volunteers is relatively low. $t$ test shows that the difference has statistical significance $(\mathrm{P}<0.001)$, the data are listed in Table 2.

Table 2. Comparison of results obtained by self-assessment of healthy volunteers and norm data $(\bar{x} \pm S D)$.

\begin{tabular}{llll}
\hline Item & Healthy volunteers $(\mathbf{n = 2 0 0})$ & Norm $(\mathbf{n = 1 3 8 8})$ & $\mathbf{P}$ \\
\hline Somatization & $1.13 \pm 0.13$ & $1.37 \pm 0.48$ & 0.000 \\
\hline $\begin{array}{l}\text { Compulsive } \\
\text { symptom }\end{array}$ & $1.29 \pm 0.27$ & $1.62 \pm 0.52$ & 0.000 \\
\hline $\begin{array}{l}\text { Interpersonal } \\
\text { sensitivity }\end{array}$ & $1.31 \pm 0.21$ & $1.65 \pm 0.61$ & 0.000 \\
\hline Depression & $1.26 \pm 0.33$ & $1.50 \pm 0.59$ & 0.000 \\
\hline Anxiety & $1.21 \pm 0.21$ & $1.39 \pm 0.43$ & 0.000 \\
\hline Hostility & $1.08 \pm 0.26$ & $1.46 \pm 0.55$ & 0.000 \\
\hline $\begin{array}{l}\text { Phobia } \\
\text { Paranoid }\end{array}$ & $1.05 \pm 0.18$ & $1.23 \pm 0.41$ & 0.000 \\
\hline $\begin{array}{l}\text { Psychotic } \\
\text { symptom }\end{array}$ & $1.17 \pm 0.26$ & $1.43 \pm 0.57$ & 0.000 \\
\hline $\begin{array}{l}\text { Average } \\
\text { positive factors }\end{array}$ & $10.32 \pm 14.26$ & $1.29 \pm 0.42$ & 0.000 \\
\hline
\end{tabular}

\section{EPQ scores}

According to EPQ results, $\mathrm{P}$ score is $4.59 \pm 2.33$, E score is $13.13 \pm 4.32, \mathrm{~N}$ score is $6.89 \pm 5.26$, and $\mathrm{L}$ score is $13.21 \pm$ 4.25 for 200 healthy volunteers. Comparing the norm in China, the $\mathrm{P}$ and $\mathrm{N}$ scores are relatively lower, and the $\mathrm{E}$ and $\mathrm{L}$ scores are relatively higher. t-test shows that the difference has statistical significance $(\mathrm{P}<0.001)$, the data are listed in Table 3 .

Table 3. Comparison of $E P Q$ results obtained by healthy volunteers and norm data $(\bar{x} \pm S D)$.

\begin{tabular}{llll}
\hline Item & Healthy volunteers $(\mathbf{n = 2 0 0})$ & Norm $(\mathbf{n = 4 5 8})$ & $\mathbf{P}$ \\
\hline P score & $4.59 \pm 2.33$ & $7.86 \pm 3.05$ & 0.000 \\
\hline E score & $13.13 \pm 4.32$ & $9.85 \pm 4.36$ & 0.000 \\
\hline N score & $6.89 \pm 5.26$ & $10.81 \pm 4.45$ & 0.000 \\
\hline
\end{tabular}

\begin{tabular}{llll}
\hline L score & $13.21 \pm 4.25$ & $11.75 \pm 3.55$ & 0.000 \\
\hline
\end{tabular}

\section{Discussion}

The adverse events collection such as SCL-90 is one of the most famous test scale for psychological health all over the world. In this method, ten factors are selected to reflect the psychological symptoms of a person, including somatization, compulsive symptom, interpersonal sensitivity, depression, anxiety, hostility, phobia, paranoid, psychotic symptom, etc. It is commonly used for psychiatric and psychological counselling clinics to understand the psychological status of counsellors, and to examine the mental problems among people having different occupations. Previous studies have shown that the questionnaire has good reliability and validity [7]. SCL-90 has been widely used domestically and internationally in high school students [8], college students $[9,10]$, soldiers [11], tumor patients [12] and other groups of people.

This study has demonstrated that the score of each factor in SCL-90 is lower than norm data of Chinese adults in 1986 [13], suggesting that the overall level of psychological health of Chinese healthy volunteers is significantly lower than general adults. This result is closely related to low-salary income, unstable employment, and large life pressure.

Relevant studies have shown that psychological status of volunteers in Phase I clinical trial mainly includes phobia, anxiety and unstable emotion [13]. Due to the lack of correct understanding towards experimental drugs and programs, Phase I volunteers might exhibit suspection and phobia to drugs and adverse events, respectively, as well as a resistance to blood sampling [14]. These observations are consistent with the results in this study. Jiang et al. [15] applied the self-rating anxiety scale and self-rating depression scale to assess psychological status of 35 healthy volunteers before and after clinical trials. They have found that healthy volunteers have anxiety before and after clinical trials. The current study shows that there are no symptoms of anxiety, phobia, somatization and paranoid found in healthy volunteers, which might be related to two aspects. First, these volunteers have participated in Phase I clinical trials before. Second, they are not currently present in a clinical trial.

During a phase I clinical trial, the drugs are used in human body for the first time. Therefore it is inevitably for the healthy volunteers to product fluctuation in their psychological conditions. This fluctuation will affect final result on the one hand [16], and result in an unsatisfied emotion on the other hand [17]. In this study, the items such as somatization, compulsive symptom, anxiety, paranoid and psychotic symptom in SCL-90 can reflect a subjective sense for physical discomfort, and trigger physical characterizations which are related to adverse events. Other factors such as interpersonal sensitivity, depression, hostility and phobia mainly reflected to the aspect of individual thinking, which related to the compliance during the process of clinical trial. Therefore, understanding the emotional characteristics of volunteers 
before phase I clinical trials is critical to the management of volunteers and adverse events during phase I clinical trial, which further affect the quality and outcome of overall clinical trial.

The EPQ describes the personality of volunteers from three mutually orthogonal dimensions, including introversion and extroversion (E), neuroticism (N), and psychoticism (P). Researchers can understand different personal characteristics from these three aspects. In this study, the adult questionnaire containing 88 questions made by Gong [6] was applied to investigate personality characteristics of healthy volunteers. The results from this study will be helpful to the research of the effects of psychological emotion on clinical results of volunteers in phase I clinical trials.

This study has demonstrated that the score of $\mathrm{E}$ factor of healthy volunteers is higher than the norm data, suggesting that the volunteers in this experimental group are more extroverted with a tendency of more outgoing personality, better social skills, more eager to experience excitement and adventure. They belong to the type of incontrollable emotion with higher social immature level. While the scores of $\mathrm{P}$ and $\mathrm{N}$ dimensional factors are lower than the norm data, suggesting that the volunteer in this group are more stable, sympathetic and concerned about others. This result is consistent with Ouyang et al.'s conclusion on 119 healthy volunteers [18], but slightly different from Jiang et al.'s paper about 35 healthy volunteers in phase I clinical trial [15]. This might due to the difference in the representative of samples. In the study of Jiang et al., the age of healthy volunteers is mainly between 18 and $22 \mathrm{y}$ old with greater emotional fluctuation. The score of $\mathrm{L}$ factor in this study indicates that current study has better stability.

Relevant studies [19] have demonstrated that the personal characteristics of the volunteers may influence pharmacokinetics or pharmacodynamics of a drug. According to Meyer et al.'s result [20], a single dose of $0.3 \mathrm{mg} / \mathrm{kg}$ diazepam which was used to an extrovert individual could significantly damage the reaction capacity, while the reaction ability of an introvert was not affected by the same amount of dose. Nakano et al.'s study showed that the oral intake of caffeine and diazepam is significantly faster to the individuals with higher score of $\mathrm{N}$ dimensional factor [21]. The current study suggests that assessment of personality characteristics of healthy volunteers is significant to the outcome of clinical trials.

This study has shown that the personality characteristics and emotional qualities of healthy volunteers are both different with Chinese norm data, and both factors will significantly affect the management and results of during the process of clinical trials. Current study suggests that valid assessment methods should be used to predict the emotional qualities and personality characteristics of the volunteers in the future before they are involved the experimental groups of clinical trials, which can provide theoretical fundamentals for the management of volunteers and test results. During the clinical trails the different emotions and personality characteristics of volunteers can be effectively administrated to improve compliance of volunteers and to minimize the occurrence of false or adverse events. The quality and efficiency of clinical trials will be further improved consequently.

This study selected a single clinical unit to conduct the research, therefore the result will contain a bias and deviation. In the future, this type of clinical trial can be carried out in multi clinical centers to minimize the selective bias and to allow the results more representative and instructive. It should be noted that the survey method used in this study are not suitable for quantitative analysis and multi-factor analysis to relevant factors. A specific method for measurement should be formulated and evaluated to access the cognition and willingness to participate in clinical trials, which will significantly facilitate the comparison between different studies.

\section{References}

1. Quality control specification for clinical trials of drugs. China Food Drug Admin 2003.

2. Fan D. Classification and phase of clinical trial. China Prescript Med 2009, 8: 48-49.

3. Liang D. Phase I clinical trial of new drugs. West China Med J 1993, 2: 151-152.

4. Chen $\mathrm{S}, \mathrm{Li}$ L. Comparison of reliability and validity between SCL-90 and norms. Chinese J Neuropsych Dis 2003, 5: 323-327.

5. Qian M, Wu G, Zhu R. Eysenck Personality Questionnaire Revised Scale for Chinese version (EPQ-RSC). J Psychol 2000, 3: 317-323.

6. Gong Y. Eysenck personality questionnaire-a revised version for China. J Psychol Sci 1984, 4: 13-20.

7. Carlozzi NE, Long PJ. Reliability and validity of the SCL-90-R PTSD subscale. J Interp Viol 2008; 23: 1162-1176.

8. Fan H, Zhang J. Analysis of SCL-90 results for high school students over the past ten years. Psychol Sci 2005, 6: 146-148.

9. Yang M. Investigation and analysis of EPQ and SCL-90 for 221 college students. Henan J Prev Med 2006; 2: 77-78.

10. Huang Y, Li L. Meta-analysis of psychological health status of Chinese college students using symptom checklist 90 (SCL-90), Chinese J Psychol Health 2009; 5: 366-371.

11. Zhang K, Yang H, Shang M. Investigation and analysis of EPQ and SCL-90 for 722 new soldiers. J Health Psychol 2008; 7: 813-815.

12. Huang Z, Guo J, Chen R. Analysis of personality, behavior and psychological characteristics in patients with digestive tract cancer. Chinese Gene Med 2006; 9: 734-736.

13. Tian L, Wang S, Gao X. Psychological investigation and intervention of volunteers before participation of clinical trials. Hebei Med J 2009; 13: 1674-1675.

14. Wang Z, Gao H, Yang H. Development of a new phase I clinical study for healthy volunteers. J Changchun Univ Trad Chinese Med 2013; 5: 924-926. 
Study of psychological health of healthy volunteers by symptom checklist 90 (SCL-90) and eysenck personality questionnaire $(E P Q)$

15. Jiang Z, Zhu Y, Mo Y. The anxiety and depression levels of healthy volunteers before and after participation of clinical trials. Chinese Pathophysiol 2008; 1: 51-53.

16. Liao S, Yang W. Effect of psychological factors on eventrelated potential accuracy of lie detection. J Clin Electroencephal 1996; 2: 76-79.

17. Ding S, Huang Y. Qualitative study of psychological experience in patients for anti-tumor drug clinical trials. Mod Hosp 2008; 7: 87-88.

18. Ouyang D, Yi S, Sun J. Personality characteristics of healthy volunteers. Journal of Clin Psychol 1997; 1: 26-28.

19. Dimascio A, Barrett JE. Comparative effects of oxazepam in high and low anxious student volunteers. Psychosomatics 1965; 6: 298-302.

20. Meyer FP, Haschke R, Rohl FW. The healthy volunteer in clinical pharmacology: personality and motivation. Eur $\mathrm{J}$ Clin Pharmacol 1995; 48: 91-96.
21. Nakano S, Ogawa N, Kawazu Y. Influence of neuroticism on oral absorption of diazepam. Clin Pharmacol Ther 1980; 27: 370-374.

\section{*Correspondence to}

Yudong Wei

Drug Clinical Trial Center

Peking University Third Hospital

PR China

Yumei Sun

Peking University School of Nursing

PR China 